Vol 11, Issue 11, 2018

\title{
NONANTIBIOTICS ENHANCE THE ANTIBACTERIAL ACTIVITY OF CEFTRIAXONE AGAINST METHICILLIN-RESISTANT STAPHYLOCOCCUS AUREUS
}

\author{
AKILANDESWARI KRISHNAN*, RUCKMANI KANDASAMY \\ Department of Pharmaceutical Technology, Bharathidasan Institute of Technology, Anna University, Tiruchirappalli - 620 024, Tamil Nadu, \\ India. Email: akilakrishna79@gmail.com
}

Received: 30 June 2018, Revised and Accepted: 25 July 2018

\section{ABSTRACT}

Objectives: Antibiotic resistance is one of the most persistent issues worldwide nowadays, and methicillin-resistant Staphylococcus aureus (MRSA) infection is one such issue where the standard therapeutic procedures involving powerful antibiotics have failed in controlling the infection.

Methods: In the present study, the antibacterial potency of the nonantibiotics troxipide (TR), mebeverine hydrochloride (Hcl), and their combinations with ceftriaxone (CEF) against MRSA has been investigated using microbiological assays of microplate dilution method and combination index interpretations of the nonantibiotics with $\beta$-lactam antibiotic CEF and the zone of inhibition method.

Results: The nonantibiotics ME and TR inhibited resistant strain tested in vitro in the checkerboard assay, where the results showed that CEF and TR exhibited minimum inhibitory concentration (MIC) at concentrations of $50 \mu \mathrm{g} / \mathrm{ml}$ and $318 \mu \mathrm{g} / \mathrm{ml}$, respectively. Interestingly, CEF when combined with TR reduced the MIC to $8 \mu \mathrm{g} / \mathrm{ml}$ and $78 \mu \mathrm{g} / \mathrm{ml}$. According to the results, CEF with TR exhibited synergistic interactions at the fractional inhibitory concentration of 0.36-1.4. ME and TR and its combinations, CEF with ME, and CEF with TR have considerable anti-MRSA efficacy, with synergism though at $36 \mathrm{~h}$ of incubation.

Conclusion: ME and TR being antispasmodic and antiulcer drugs can also be used against MRSA infections, which could prove to be favorable in the reduction of dosage of antibiotics such as CEF, and cutting down the need for additional administration of antibiotics to the patients affected with multiple complications such as gastrointestinal ulcer, spasm difficulties, and infection.

Keywords: Antibacterial activity, Ceftriaxone, Combination index interpretation, MebeverineHcl, Troxipide.

(C) 2018 The Authors. Published by Innovare Academic Sciences Pvt Ltd. This is an open access article under the CC BY license (http://creativecommons. org/licenses/by/4. 0/) DOI: http://dx.doi.org/10.22159/ajpcr.2018.v11i11.21535

\section{INTRODUCTION}

Resistance to antibiotics occurs when an antibiotic loses its ability to kill or effectively controls the growth of bacteria. Every time, an antibiotic is taken the sensitive strains of bacteria gets killed and the resistant strain survives, which can rapidly multiply and cause serious infections. Inappropriate and prolonged use of antibiotics can actually accelerate the emergence of resistant bacterial strains [1]. The increase and spread of drug resistance over the past 50 years have led to the distribution of resistance genes among pathogenic microorganism [2]. Infections related to antibiotic resistance were initially observed in hospital settings among critically ill and immunocompromised patients. Recent studies show that these infections are reported to be increasing in public and certain instances, in live-stock animals $[3,4]$, which makes it clear that infection is something which is contradictory to the good things in life: It is easy to get but hard to lose. Without effective treatment, the standard medical procedures will stand to fail or turn into high-risk procedures. According to the world health organization, antibiotic resistance in the pathogenic bacteria causing common infections is of high proportions (for instance, pneumonia, urinary tract infection, or blood infections) worldwide? Many bacterial infections caused by $S$. aureus, Klebsiella pneumoniae, Enterobacter cloacae, Escherichia coli, Enterococcus faecalis, etc., are treatable using antibiotics; however, with the development of resistance to multiple drugs of these bacterial strains, the general procedure of treatment has become complicated [5].

Under such circumstance, the exploration of alternative therapeutic agents that can fight against severe bacterial infections was crucial, and nonantibiotic agents that can confer antibacterial activity were brought into focus. Systematic searching from the mid-1960s, notably by many researchers gave real evidences of the fact that many eukaryotes-directed drugs antihistamine, antipsychotic, antihypertensive, antispasmodic, and anti-inflammatory have antimicrobial and antivirulence properties [6,7]. Such pharmaceutical compounds basically developed as drugs to treat noninfectious diseases which, on the other hand, have antimicrobial activities are called nonantibiotics [8]. In the present study, we have evaluated the antibacterial activity of antiulcer drug troxipide (TR) and antispasmodic drug mebeverine Hcl (ME) in vitro and discovered synergism with antibiotic ceftriaxone (CEF) against methicillinresistant Staphylococcus aureus (MRSA).
Troxipide<smiles>COc1cc(C(=O)NC2CCCNC2)cc(OC)c1OC</smiles>

Mebeverine Hcl<smiles>CCN(CCCCOC(=O)c1ccc(OC)c(OC)c1)C(C)Cc1ccc(OC)cc1</smiles>

$\mathrm{HCl}$
Ceftriaxone<smiles>CN=C(NC(=O)C1S[C@@H]2SCC(C(=O)O)=C(CSc3nc(=O)c(=O)[nH]n3C)C(=O)N12)c1csc(N)n1</smiles> 


\section{MATERIALS AND METHODS}

\section{Bacterial strains}

Clinical strain Staphylococcus aureus was obtained from the KAP Viswanathan Medical College, Tiruchirappalli, Tamil Nadu, India. The MRSA strain was confirmed and stored at $4^{\circ} \mathrm{C}$ until use.

\section{Drugs}

CEF, TR, and ME were obtained as pure drug powders from Sigma Aldrich, India, and kept under refrigeration until use.

\section{Media}

Mueller-Hinton broth (MHB) and Mueller-Hinton agar (MHA) were prepared and steam sterilized at $15 \mathrm{psi}$ for $15 \mathrm{~min}$ by autoclaving.

\section{Preparation of inocula}

The $S$. aureus was grown in MHB overnight at $37^{\circ} \mathrm{C}$ in an incubator under standard conditions. The harvested cells in the exponential phase culture were suspended in sterile distilled water and adjusted to a turbidity of $0.5 \mathrm{McFarland}$ standard using a spectrophotometer (Cary60 UV-Visible, Agilent Technologies) at $600 \mathrm{~nm}$.

\section{Checkerboard methods for studying interaction between antibiotics and nonantibiotics}

To determine the antibacterial efficacy between the individual and different combination of drugs, the following combinations, namely $\mathrm{CEF}+\mathrm{TR} ; \mathrm{CEF}+\mathrm{ME}$ and the clinical strain of MRSA have been studied in the following assay using the checkerboard method. Aliquots of log phase bacterial culture (turbidity of 0.5 McFarland standard) were transferred to microtiter plates containing tested concentrations of drugs as inferred from the minimum inhibitory concentration (MIC) assay $[9,10]$. The combination of different concentrations was used in the study. In the first row, only CEF and in the first column only TR or ME were distributed in microwell plates. CEF at $0.125-32 \mu \mathrm{g} / \mathrm{ml}$; TR at $6.25-200 \mu \mathrm{g} / \mathrm{ml}$; and ME at $1.56-100 \mu \mathrm{g} / \mathrm{ml}$ used individually. Whereas, in combination CEF + TR $(1: 10)-0.25-8 \mu \mathrm{g} / \mathrm{ml}$ of CEF with $2.5-80 \mu \mathrm{g} / \mathrm{ml}$ of TR, CEF + ME $(1: 10)-0.25-8 \mu \mathrm{g} / \mathrm{ml}$ of CEF with 2.5$80 \mu \mathrm{g} / \mathrm{ml}$ of ME. Dilute an overnight culture of MRSA in Mueller-Hinton broth to achieve optical density (OD) based inoculum of $1 \times 10^{5}-5 \times 10^{5}$. Inoculate all wells (except the column specified which is considered as blank) with $50 \mu \mathrm{l}$ of the diluted organism (the volume in each well should be $200 \mu \mathrm{l}$ ). The inoculated microtiter plates were incubated at $37^{\circ} \mathrm{C}$ for $36 \mathrm{~h}$. The growth inhibition was measured by determining the absorbance at $600 \mathrm{~nm}$ in a multimode microplate reader (Enspire, Perkin Elmer). From these readings, the synergistic and additive interactions were calculated [11-14].

The synergistic combination index (CI) was calculated in the isobologram software. The combination of the two test samples was graphically represented by dose-effect curve, CI value, dose-reduction index (DRI), and the isobole method which was interpreted in the isobologram software.

Dose effect was calculated from the following formula:

Absorbance of positive control (without drug) -

Absorbance of test solution

Absorbance of positive control

The percentage inhibition of each drug and its combination were obtained from dose-effect value multiplied by 100 .

The $\mathrm{CI}$ value of the combined drugs CEF, TR, and CEF+TR was calculated using the formula

$$
\begin{aligned}
& \mathrm{CI}=\frac{\text { MIC of substance } \mathrm{A} \text { in combination }}{\text { MIC of substance } \mathrm{A} \text { alone }}+ \\
& \frac{\text { MIC of substance } \mathrm{B} \text { in combination }}{\text { MIC of substance } \mathrm{B} \text { alone }}
\end{aligned}
$$

In the above equation substance $\mathrm{A}$ in the numerator denotes MIC of CEF in CEF+TR combination, and in denominator substance A alone indicates MIC of CEF alone; similarly, in numerator substance B means MIC of TR in combination of CEF+TR, and in denominator substance B alone means MIC of TR alone.

The goodness of fit to the straight line can be determined by the linear correlation coefficient ( $r$ value) calculated from $\mathrm{x}$ and $\mathrm{y}$ intercepts which represent

$y=a x+b, A$ plot $x=\log (D) V s y=\log (f a / f u)$, where $D$ - Dose of the drug; fa - fraction affected (bacterial growth inhibition); fu - fraction unaffected(no growth inhibition).

$f a+f u=1, f u=1-f a$.

DRI was calculated from the following equation, where $\left(D_{A}\right)_{1}$ is the dose of drug CEF alone that inhibits $\mathrm{x} \%$, $\mathrm{D}$ is the dose of the drug in combination. DRI can be really obtained from the reciprocal of each term of the $\mathrm{CI}$ equation, thus

$(\mathrm{DRI})_{1}=\left(\mathrm{D}_{\mathrm{A}}\right)_{1} /(\mathrm{D})_{1} ;(\mathrm{DRI})_{2}=\left(\mathrm{D}_{\mathrm{B}}\right)_{2} /(\mathrm{D})_{2}$

DRI $>1$ and $<1$ indicates favorable and not favorable dose reduction, respectively; DRI=1 indicates no dose reduction.

Determination of antibacterial efficacy of nonantibiotics ME and TR, and its combinations with CEF by zone of inhibition method The combination of $\mathrm{CEF}+\mathrm{TR}$ and $\mathrm{CEF}+\mathrm{ME}$ was tested by studying the zone of inhibition by using the well-diffusion technique. Plates were prepared using preinoculated (1-day-old cultures of 0.5 McFarland standard turbidity) MHA, on the surface of which four wells were prepared using gel puncture and filled with different concentrations of test agents and its combinations with respect to the MIC values. The plates were incubated at $37^{\circ} \mathrm{C}$ for $18 \mathrm{~h}$, and the zone of inhibition of MRSA was measured. The experiments were performed in triplicates, and the synergistic effects were statistically evaluated [12].

\section{RESULTS}

\section{Synergistic effect observed between CEF, TR, and CEF+TR} combination against MRSA

The bacterial growth inhibitory effect of CEF, TR, and CEF+TR on MRSA was observed and interpreted. The $\mathrm{CI}$ values of the combined drugs CEF, $\mathrm{TR}$, and CEF+TR were calculated using the above-said formula and its results summarized in Table 1. It shows the results of the checkerboard assay for the determination of MIC of the antibiotics CEF and TR and its combination of CEF+TR against the clinical strain of MRSA. CEF and TR exhibited MIC at a concentration of $50 \mu \mathrm{g} / \mathrm{ml}$ and $318 \mu \mathrm{g} / \mathrm{ml}$, respectively. Whereas, $\mathrm{CEF}+\mathrm{TR}$ as a combination has shown a significant reduction in the required effective concentration of CEF and TR which is $8 \mu \mathrm{g} / \mathrm{ml}$ and $78 \mu \mathrm{g} / \mathrm{ml}$, respectively. This shows that CEF and TR exhibit a synergistic effect when put together as a combination (CEF+TR), thereby exhibiting increased in the antibacterial potency against MRSA. The CI values of the combined drugs were calculated. According to the results, synergistic interactions were derived for CEF+TR (0.36-1.4).

From the above-said formula, growth affected (fa) and growth unaffected (fu) were calculated, and the dose-effect curve was plotted using the isobologram software. Graphical representation of a doseeffect curve, a logarithmic CI, a DRI, and isobologram for each test sample and its combination have been presented below in Fig. 1a-d. The dose-effect curve was plotted using optical density (OD) value in Fig. 1a, with the help of the curve $\mathrm{x}$ intercept, $\mathrm{y}$ intercept, and the median effect of the dose was calculated. Logarithmic CI plot for CEF+TR indicated in Fig. 1b, where CI point lying between the diagonal line indicates synergistic effects and DRI of the combination shown in Fig. 1c, DRI>1 indicates a favorable dose reduction.

Isobologram used to interpret $50 \%, 75 \%$, and $90 \%$ inhibition is shown in Fig. 1d. Results revealed in the CEF+TR combination, data point 
(values) on the isobologram, where lower left indicates synergistic effect. From the above observations, the MIC of CEF showed sensitivity at certain concentrations; whereas, its effect was enhanced even in low concentrations when the drug was used in combination.

\section{Synergistic effect observed between CEF, ME and CEF+ME combination against MRSA}

The bacterial growth inhibitory effect of CEF, ME, and CEF+ME on MRSA was observed and interpreted. The $\mathrm{CI}$ values of the combined drugs $\mathrm{CEF}, \mathrm{ME}$, and $\mathrm{CEF}+\mathrm{ME}$ were calculated using the above-mentioned formula and its results represented in Table 2. It shows the results of the checkerboard assay for the determination of MIC of the antibiotics $\mathrm{CEF}$ and $\mathrm{ME}$ and its combination $\mathrm{CEF}+\mathrm{ME}$ against the clinical strain of MRSA. CEF and ME exhibited MIC at the concentration of $50 \mu \mathrm{g} / \mathrm{ml}$ and $80 \mu \mathrm{g} / \mathrm{ml}$, respectively. Whereas, $\mathrm{CEF}+\mathrm{ME}$ as a combination has shown a slight increased reduction in the required effective concentration of CEF and ME which is $10.59 \mu \mathrm{g} / \mathrm{ml}$ and $78 \mu \mathrm{g} / \mathrm{ml}$, respectively. This shows that CEF and ME exhibits a lesser synergistic effect when put together as a combination (CEF+ME), thereby exhibiting only a slight increase in the antibacterial potency against MRSA. The CI value of the combined drugs was calculated, and it is not under the CI value below 1.5. According to the results, lesser synergistic interactions were derived for CEF + ME (1.0-1.67).

Graphical representation of a dose-effect curve, logarithmic CI, DRI, and isobologram for each test sample and its combination has been presented in Fig. 2a-d. The dose-effect curve was plotted using the OD value in Fig. $2 \mathrm{a}$, with the help of the curve $\mathrm{x}$ intercept, $\mathrm{y}$ intercept, and the median effect of the dose was calculated. Logarithmic CI plot for $\mathrm{CEF}+\mathrm{ME}$ shown in Fig. $2 \mathrm{~b}$, where CI point lying on the diagonal line indicates synergistic effects and DRI of the combination represented in Fig. 2c, DRI $<1$ indicates nearly favorable dose reduction. Isobolograms for 50\%, 75\%, and 90\% inhibition are shown in Fig. $2 \mathrm{~d}$. Combination data point on the lower left indicates synergism. In the present combination of $\mathrm{CEF}+\mathrm{ME}, \mathrm{IC}_{75}$, and $\mathrm{IC}_{50}$ showed the nearly less synergistic effect.

\section{Synergistic effect confirmed in zone of inhibition method}

In Table 3, the disc diffusion tests demonstrated the inhibitory effects of CEF, TR, and ME individually inhibited the bacterial growth $25.3 \mathrm{~mm}$, $16 \mathrm{~mm}$, and $16 \mathrm{~mm}$, respectively; whereas, $\mathrm{CEF}+\mathrm{TR}$ and CEF+ME against MRSA show an inhibitory zone of $43 \mathrm{~mm}$ and $41 \mathrm{~mm}$, respectively. Thus, the synergistic effects of $\mathrm{CEF}+\mathrm{TR}$ and $\mathrm{CEF}+\mathrm{ME}$ combined drugs were observed to some extent.

\section{DISCUSSION}

Identification of mecA gene in clinical sample of $S$. aureus has been confirmed in earlier literature [13]. The presence of mecA gene in the clinical sample confirmed the MRSA strain through which it is made sure that the working strain is MRSA. TR, 3-(3,4,5-trimethoxy benzamide) piperidine, is used in the treatment of gastroesophageal reflux disease and ME, 4-[ethyl-[1-(4-methoxy phenyl) propan-2-yl]amino]butyl 3,4-dimethoxy benzoate hydrochloride, is used in the management of irritable bowel syndrome and abdominal cramping. Results from checkerboard assay for the determination of MIC of the antibiotics CEF, ME, and TR and its combinations AM + ME, CEF + ME, and CEF + TR against the clinical strain of MRSA show that the test agents under combination exhibit a visible synergistic effect. Based on the results, CEF $+\mathrm{TR}$ as a combination shows a significant $1 / 6^{\text {th }}$ and $1 / 5^{\text {th }}$ reduction in the required effective concentration of $\mathrm{CEF}$ and $\mathrm{TR}$ with $\mathrm{CI}$ ranging from 0.36 to 1.4 , whereas, $\mathrm{CEF}+\mathrm{ME}$ as a combination shows $1 / 5^{\text {th }}$ reduction in the required effective concentration of CEF; however, there is a visible similarity in the required effective concentration of $\mathrm{ME}$ with $\mathrm{CI}$ ranging from 1.0 to 1.6 collectively. The major aim for achieving synergy in drug combination is that it allows dose-reduction for the good therapeutic effect. The beneficial significance of dose reduction is the reduced toxicity toward the host. DRI (DRI) denotes how many fold of dose reduction is allowed for each drug due to synergism when compared with the dose of each drug alone. From the above investigation, it is inferred that the combination of antibiotics and nonantibiotics CEF+TR having $\mathrm{CI}>0.5$ has a synergistic effect as any combination having $\mathrm{CI}<0.5$ only is considered to have strong synergistic effect. Unlike the above combinations, as CEF + ME shows less decrease in the MIC of ME, the combination is considered as favorable even though at very lowdose reduction. Although CEF+TR combination projects a synergistic effect, the combination does exhibit MRSA growth inhibition; hence, this combination of drugs can be further studied with different concentrations for strong and improved anti-MRSA activity.

Disc-diffusion test has confirmed the inhibitory effects of CEF, TR, and ME individually with bacterial growth inhibited up to $25.3 \mathrm{~mm}, 16 \mathrm{~mm}$, and $16 \mathrm{~mm}$, respectively; whereas, $\mathrm{CEF}+\mathrm{TR}$ and $\mathrm{CEF}+\mathrm{ME}$ show an inhibitory zone of $43 \mathrm{~mm}$ and $41 \mathrm{~mm}$, respectively. Therefore, the visible synergistic effect of combined drugs could be observed to some extent with better antimicrobial efficacy compared to that of individual test samples. However, both TR and ME growth inhibitions were achieved after $36 \mathrm{~h}$, where the duration of action is longer when compared with that of the conventional antibiotics $(18 \mathrm{~h})$.

Table 1: Checkerboard method for non-antibiotics CEF, TR, and CEF+TR

\begin{tabular}{|c|c|c|c|c|c|c|c|c|c|}
\hline \multirow{3}{*}{$\begin{array}{l}\text { Test agents }(\mu \mathrm{g} / \mathrm{ml} \\
\mathrm{CEF}\end{array}$} & \multicolumn{3}{|c|}{ Growth inhibition $50 \%$} & \multicolumn{3}{|c|}{ Growth inhibition $75 \%$} & \multicolumn{3}{|c|}{ Growth inhibition $90 \%$} \\
\hline & \multirow{2}{*}{ CI value } & \multicolumn{2}{|c|}{ Conc. $\mu g / m l$} & \multirow{2}{*}{ CI value } & \multicolumn{2}{|c|}{ Conc. $\mu \mathrm{g} / \mathrm{ml}$} & \multirow{2}{*}{ CI value } & \multicolumn{2}{|c|}{ Conc. $\mu \mathrm{g} / \mathrm{ml}$} \\
\hline & & 1.04 & - & & 7.19 & - & & 49.76 & - \\
\hline $\mathrm{TR}$ & - & - & 78.05 & - & - & 171.87 & - & - & 318.61 \\
\hline $\mathrm{CEF}+\mathrm{TR}$ & 1.45 & 1.33 & 13.27 & 0.63 & 3.22 & 32.18 & 0.36 & 7.8 & 78.02 \\
\hline
\end{tabular}

All the values represent triplicate values. $\mathrm{CI}<1$ indicates synergism, $\mathrm{CI}>1$ indicates additive effect; $\mathrm{CI}<0.5$, synergy; $\mathrm{CI} 0.5-0.75$ partial synergy. $\mathrm{CEF}$ and TR exhibited MIC at 49.8, 318.6, respectively, when CEF combines with TR the dose reduced to $1 / 6$ of the MIC of CEF ( $7.8 \mu \mathrm{g} / \mathrm{ml})$ and $1 / 5$ of the MIC of TR (78 $\mu \mathrm{g} / \mathrm{ml}) \mathrm{respectively.}$ CEF: Ceftriaxone, TR: Troxipide, CEF+TR: Ceftriaxone with troxipide, ME: MebeverineHcl, CI: Combination index, MIC: Minimum inhibitory concentration

Table 2: Checkerboard method for nonantibiotics CEF, ME, and CEF+ME

\begin{tabular}{|c|c|c|c|c|c|c|c|c|c|}
\hline \multirow[t]{2}{*}{ Test agents $(\mu \mathrm{g} / \mathrm{ml}$} & \multicolumn{3}{|c|}{ Growth inhibition $50 \%$} & \multicolumn{3}{|c|}{ Growth inhibition $75 \%$} & \multicolumn{3}{|c|}{ Growth inhibition $90 \%$} \\
\hline & \multirow{2}{*}{$\begin{array}{l}\text { CI value } \\
-\end{array}$} & \multicolumn{2}{|c|}{ Conc. $\mu \mathrm{g} / \mathrm{ml}$} & \multirow{2}{*}{$\begin{array}{l}\text { CI value } \\
-\end{array}$} & \multicolumn{2}{|c|}{ Conc. $\mu \mathrm{g} / \mathrm{ml}$} & \multirow{2}{*}{$\begin{array}{l}\text { CI value } \\
-\end{array}$} & \multicolumn{2}{|c|}{ Conc. $\mu \mathrm{g} / \mathrm{ml}$} \\
\hline CEF & & 1.04 & - & & 7.19 & - & & 49.76 & - \\
\hline ME & - & - & 28.99 & - & - & 48.08 & - & - & 79.76 \\
\hline $\mathrm{CEF}+\mathrm{ME}$ & 1.67 & 1.28 & 12.8 & 1.28 & 3.68 & 36.82 & 1.54 & 10.59 & 78.94 \\
\hline
\end{tabular}

All the values represent triplicate values. $\mathrm{CI}<1$ indicates synergism, $\mathrm{CI}>1$ indicates additive effect, $\mathrm{CI}<0.5$, synergy; $\mathrm{CI} 0.5-0.75$, partial synergy. CEF and $\mathrm{ME}$ individually exhibited MIC at $50,80 \mu \mathrm{g} / \mathrm{ml}$, respectively, when it was combined with ME dose reduced to $1 / 5$ of the MIC of CEF whereas ME dose same as that of MIC of ME.

CI: Combination index, CEF: Ceftriaxone, ME: MebeverineHcl, MIC: Minimum inhibitory concentration 


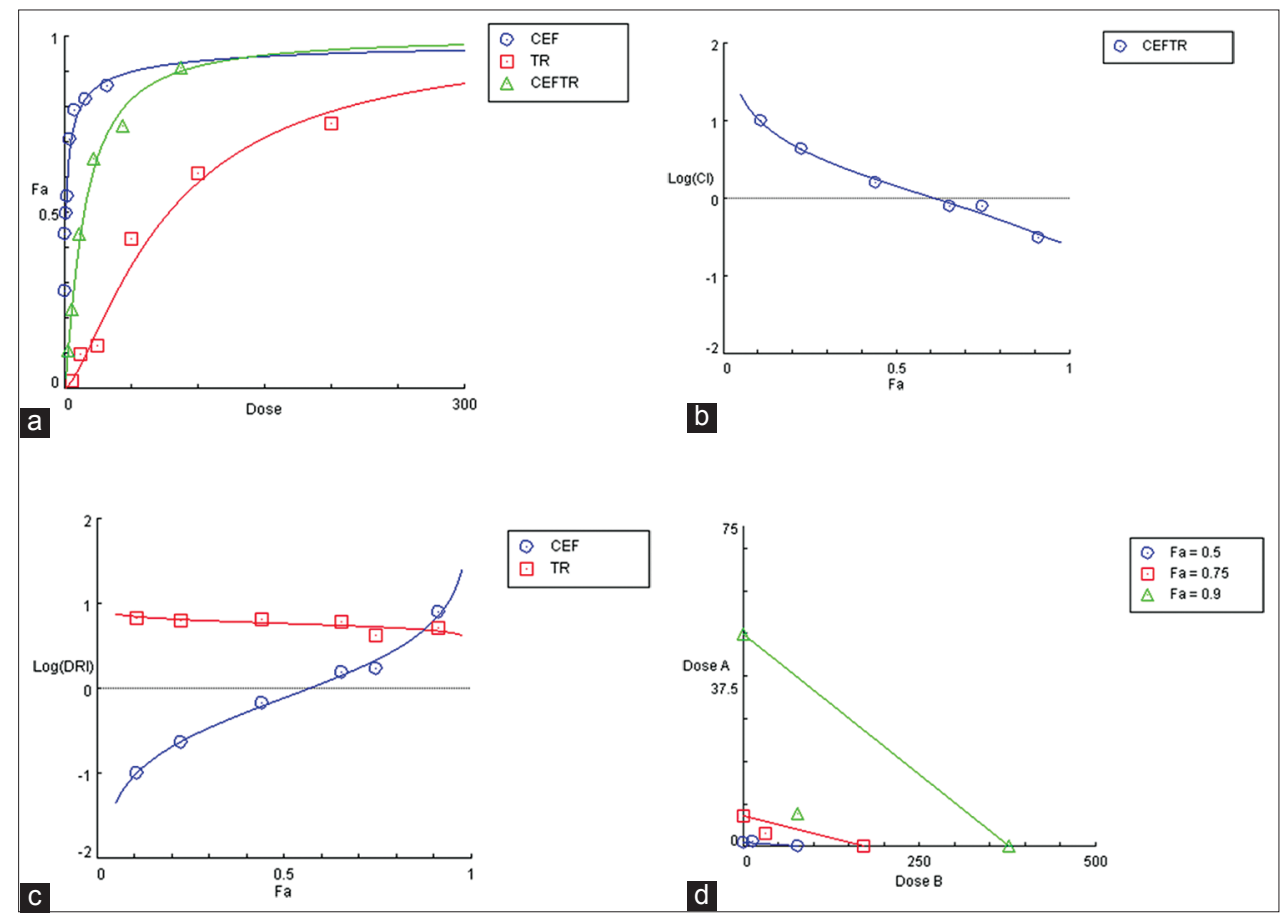

Fig. 1: CI determination for CEF with TR against MRSA. (a) Dose-effect curve for CEF, TR, and CEF+TR. (b) Logarithmic combination index plot for CEF, TR, and CEF+TR. Log (CI): Logarithmic combination index. (c) Logarithmic dose-reduction index plot for combination CEF+TR [1:10]. (d) Isobologram for combination CEF+TR [1:10]. Fa: Fraction affected (growth inhibited), CEF: Ceftriaxone, TR: Troxipide, CEFTR: Ceftriaxone with troxipide, Log (DRI): Logarithmic dose reduction index, CI: Combination index, Fa $=0.75$ indicates $\mathbf{7 5 \%}$ inhibition, Fa $=0.9$ indicates $90 \%$ inhibition, Dose A indicates ceftriaxone, Dose B indicates troxipide, $\mathrm{Fa}=0.5$ indicates $50 \%$ inhibition, MRSA: Methicillin resistant Staphylococcus aureus

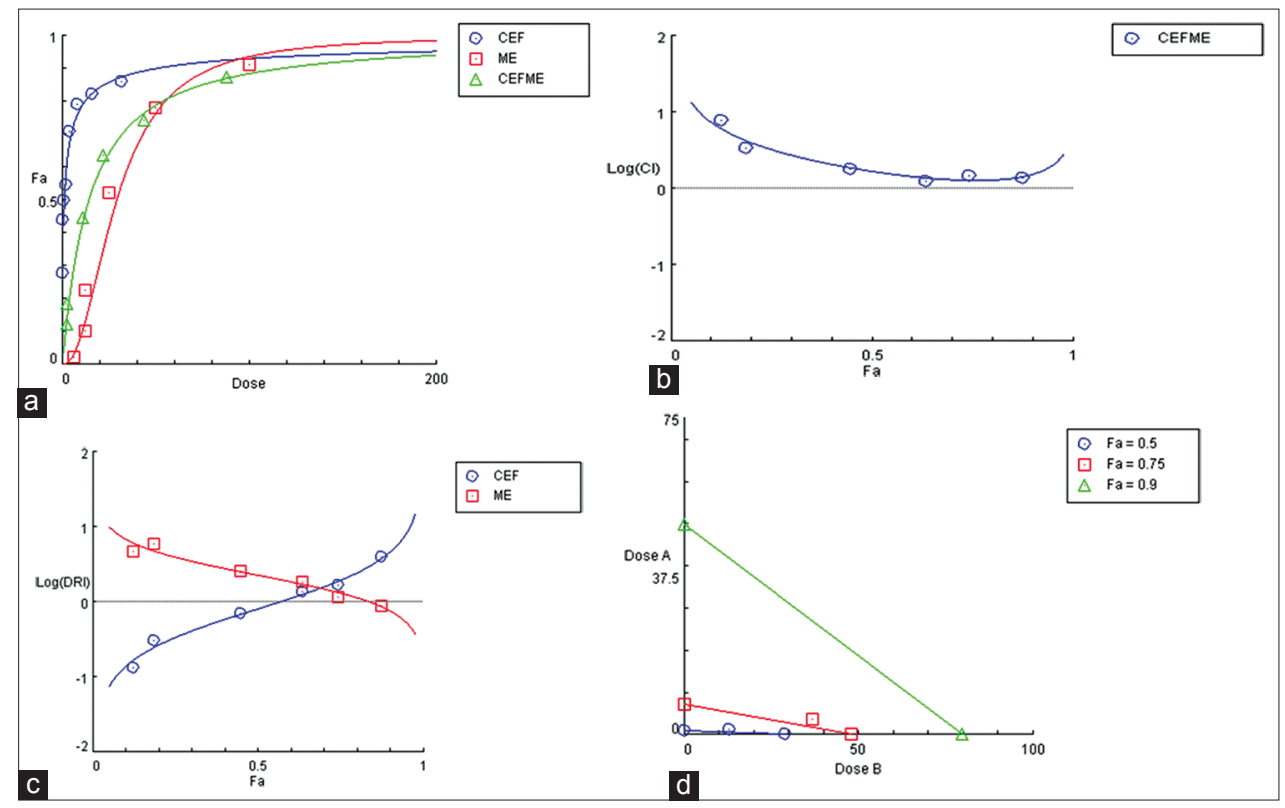

Fig. 2: CI determination for CEF with ME against MRSA. (a) Dose-effect curve for CEF, ME, and CEF+ME. (b) Logarithmic combination index plot for CEF, ME, and CEF+ME. (c) Log (DRI) plot for combo: CEFME (CEF+ME [1:10]). (d) Isobologram for Combo: CEFME (CEF+ME [1:10]). Fa: Fraction affected (growth inhibited), CEF: Ceftriaxone, TR: Troxipide, CEFTR: Ceftriaxone with troxipide. Log (CI): Logarithmic combination index, CEFME: Ceftriaxone with mebeverine hydrochloride, Log (DRI): Logarithmic dose reduction index, ME: Mebeverine hydrochloride, Dose A indicates ceftriaxone, Dose B indicated MebeverineHcl, Fa=0.5 indicates 50\% inhibition, $\mathrm{Fa}=\mathbf{0 . 7 5}$ indicates $\mathbf{7 5 \%}$ inhibition, Fa=0.9 indicates $\mathbf{9 0 \%}$ inhibition, MRSA: Methicillin resistant Staphylococcus aureus, CI: Combination index

From the discussion, it can be concluded that both the proposed nonantibiotics $\mathrm{ME}$ and TR that are commercially marketed as antispasmodic and antiulcer drugs have noticeable antibacterial efficacy. The fact that the chemical structure of ME, 3-(3,4,5-trimethobenzamide) piperidine side chain, and TR, 3-(3,4,5-trimethoxy benzamide) piperidine attributes to the antibacterial activity [15].
It has been proved from the earlier studies that antibiotics with nonantibiotics when used in combination had an enhanced antibacterial capability against Gram-positive and Gram-negative microorganisms [16]. On the basis of MIC and disc-diffusion method, the antibacterial activity of ME and TR and its following synergistic effect with an antibiotic CEF is similar to the nonantibiotics 
Table 3: Determination of the synergistic effect of the combination of CEF+TR and CEF+ME against MRSA by the zone of inhibition method

\begin{tabular}{lll}
\hline S. No & $\begin{array}{l}\text { Name of the selected drug } \\
\text { combinations } \boldsymbol{\mu g} / \mathbf{1 0} \boldsymbol{\mu l}\end{array}$ & $\begin{array}{l}\text { Zone of inhibition in } \mathbf{~ m m} \\
\text { against MRSA }\end{array}$ \\
\hline 1 & CEF (50) & $25.3 \pm 0.58$ \\
2 & TR (300) & $16 \pm 1.0$ \\
3 & ME (75) & $16 \pm 1.5$ \\
4 & CEF+TR (8+80) & $43 \pm 0.58$ \\
5 & CEF+ME (11+80) & $41 \pm 1.58$ \\
\hline
\end{tabular}

\pm indicates standard deviation of triplicates values. CEF: Ceftriaxone,

TR: Troxipide, CEF+TR: Ceftriaxone with troxipide, MRSA: Methicillin-resistant Staphylococcus aureus, ME: MebeverineHcl

diclofenac sodium [17] oxyfedrine [12] omeprazole, ranitidine [18], dicyclomine [15], azelastin [19], and synthetic derivatives [20,21]. Although the mechanism of bacterial inhibition of nonantibiotics was not well known, it could be due to multiple factors interfering with bacterial cell wall synthesis. Specifically, multiple receptors found in the MRSA strain could be responsible for the reduction [22]. These ligands might prove to be moderate inhibitors for MRSA infections, thus proving to be potentially beneficial for creating a novel antibacterial therapeutic molecule, and the compounds ME and TR were found to have antibacterial activity.

\section{CONCLUSION}

The study it is concluded that the nonantibiotics ME and TR and its combinations, with $\beta$-lactam antibiotics, CEF+TR have potency against MRSA infection, which could be a synergistic impact of the combinations. These combinations of drugs can be further studied with different concentrations for strong and improved anti-MRSA activity. ME and TR being antispasmodic and antiulcer drugs can also be used against MRSA infections which could prove to be favorable in the reduction of dosage of antibiotics such as CEF, and cutting down the need for additional administration of antibiotics to the patients affected with multiple complications such as gastrointestinal ulcer, spasm difficulties, and infection.

\section{ACKNOWLEDGMENTS}

The authors gratefully acknowledge the financial grant awarded by the Department of Science and Technology, Ministry of Science and Technology, Government of India, in the form of facility project "National facility for Drug Development for Academia, Pharmaceutical and Allied industries."

\section{CONFLICTS OF INTEREST}

The authors declare no conflicts of interest.

\section{REFERENCES}

1. Levy SB. The Antibiotic Paradox: How the Misuse of Antibiotics Destroys their Curative Powers. $2^{\text {nd }}$ ed. Cambridge, MA: Perseus Publishing, Cambridge; 2002. p. 353.

2. Martins M, Dastidar SG, Fanning S, Kristiansen JE, Molnar J, Pagès JM, et al. Potential role of non-antibiotics (helper compounds) in the treatment of multidrug-resistant gram-negative infections: Mechanisms for their direct and indirect activities. Int J Antimicrob
Agents 2008;31:198-208.

3. Alanis AJ. Resistance to antibiotics: Are we in the post-antibiotic era? Arch Med Res 2005;36:697-705.

4. Stefani S, Chung DR, Lindsay JA, Friedrich AW, Kearns AM, Westh H, et al. Meticillin-resistant Staphylococcus aureus (MRSA): Global epidemiology and harmonisation of typing methods. Int J Antimicrob Agents 2012;39:273-82

5. Christine L. Case. Discover a New Antibiotic; 1998.

6. Dutta NK, Annadurai S, Mazumdar K, Dastidar SG, Kristiansen JE, Molnar J, et al. Potential management of resistant microbial infections with a novel non-antibiotic: The anti-inflammatory drug diclofenac sodium. Int J Antimicrob Agents 2007;30:242-9.

7. Mazumdar K, Dastidar SG, Park JH, Dutta NK. The anti-inflammatory non-antibiotic helper compound diclofenac: An antibacterial drug target. Eur J Clin Microbiol Infect Dis 2009;28:881-91.

8. Kristiansen JE. Antimicrobial activity of non-antibiotics. ASM News 1991;57:135-9.

9. Busri A, Puspitawati R, Utami S. Antibacterial effect of java turmeric ethanol extract against dual-species Streptococcus mutans and Streptococcus sanguinis biofilm (in vitro). Asian J Pharm Clin Res 2017;10:57-60.

10. Sukandar EY, Kurniati NF, Wikaningtyas P, Agprikani D. Antibacterial interaction of combination of ethanolic extract of Zingiber officinale var Rubrum rhizome, Boesenbergia pandurata rhizome and Stevia rebaudiana leaves with certain antibiotics against infectious mouth microbial. Asian J Pharm Clin Res 2016;9:332-5.

11. Kumar KA, Ganguly K, Mazumdar K, Dutta NK, Dastidar SG, Chakrabarty AN, et al. Amlodipine: A cardiovascular drug with powerful antimicrobial property. Acta Microbiol Pol 2003;52:285-92.

12. Mazumdar K, Ganguly K, Kumar KA, Dutta NK, Chakrabarty AN, Dastidar SG, et al. Antimicrobial potentiality of a new non-antibiotic: The cardiovascular drug oxyfedrine hydrochloride. Microbiol Res 2003;158:259-64.

13. Eumkeb G, Chukrathok S. Synergistic activity and mechanism of action of ceftazidime and apigenin combination against ceftazidime-resistant Enterobacter cloacae. Phytomedicine 2013;20:262-9.

14. Devi KP, Nisha SA, Sakthivel R, Pandian SK. Eugenol (an essential oil of clove) acts as an antibacterial agent against salmonella typhi by disrupting the cellular membrane. J Ethnopharmacol 2010;130:107-15.

15. Karak P, Kumar KA, Mazumdar K, Mookerjee M, Dastidar SG. Antibacterial potential of an antispasmodic drug dicyclomine hydrochloride. Indian J Med Res 2003;118:192-6.

16. Akilandeswari K, Ruckmani K. Antibacterial potentiality of anti-ulcer and anti-spasmodic drugs with selected antibiotics against methicillinresistant Staphylococcus aureus - In vitro and in silico studies. Bangladesh J Pharmacol 2015;10:875-83.

17. Dutta NK, Mazumdar K, Seok SH, Park JH. The anti-inflammatory drug diclofenac retains anti-listerial activity in vivo. Lett Appl Microbiol 2008;47:106-11.

18. Alkuraishy HM. In vitro antibacterial effects of selective histaminic receptor Type 2 blockers: A novel study. Webmed Cent Pharmacol 2011;2:WMC002636.

19. El-Nakeeb MA, Abou-Shleib HM, Khalil AM, Omar HG, El-Halfawy OM. Reversal of antibiotic resistance in gram-positive bacteria by the antihistaminic azelastine. APMIS 2012;120:215-20.

20. Shafiq B, Muhi-Eldeen Z, Al-kaissi E, Al-adham IS. Synthesis, structural elucidation and antimicrobial evaluation of 2-\{4-(tamino)-2(but-2-yn-1-yl)\}-1, 3 benzothiazole derivatives. Int J Pharm PharmSci 2016;8:189-93.

21. Dilipkumar P, Vipul S, Desh DP, Roopesh KM. Synthesis, characterization and antimicrobial evaluation of some 1,2, 4- triazole derivatives. Int J Pharm Pharm Sci 2014;6:213-6.

22. Tomasz AS, Tomasz A. Role of penicillin-binding protein 2 (PBP2) in the antibiotic susceptibility and cell wall cross-linking of Staphylococcus aureus: Evidence for the cooperative functioning of PBP2, PBP4, and PBP2A. J Bacteriol 2005;5:1815-24 